\title{
Adsorption and Transport of Ciprofloxacin in Quartz Sand at Different pH and Ionic Strength
}

\author{
Xiujiao Xu*, Jianglong He, Yu Li, Zhaoxi Fang, Shaohui Xu\# \\ Department of Environment Science, Qingdao University, Qingdao, China \\ Email: “xjxuqd@sina.com, "shhxu@qdu.edu.cn
}

Received 29 September 2014; accepted 25 October 2014; accepted 8 November 2014

Copyright (C) 2014 by authors and Scientific Research Publishing Inc.

This work is licensed under the Creative Commons Attribution International License (CC BY). http://creativecommons.org/licenses/by/4.0/

(c) (i) Open Access

\section{Abstract}

Effects of pH and ionic strength on ciprofloxacin adsorption in quartz sand were studied through a batch equilibrium adsorption experiment in this paper. The experimental data were fitted by empirical formulas from Langmuir and Freundlich adsorption isothermal curves, and the transport experiments in quartz sand at different $\mathrm{pH}$ and ionic strength were conducted to investigate the transport characteristics of ciprofloxacin. It was found that with the increase of pH value or ionic strength, adsorption capacity of ciprofloxacin decreased, so that it could move easier. The results indicated that low pH or ionic strength was conductive to the adsorption of ciprofloxacin in quartz sand. Meanwhile, a higher initial concentration or stronger ionic strength could result in a smaller linear distribution coefficient of ciprofloxacin, which meant a low adsorption capacity. According to the fitting results, the adsorption of ciprofloxacin in quartz sand could be described well by both Langmuir and Freundlich equations, of which Freundlich equation had a better efficacy.

\section{Keywords}

Ciprofloxacin, Quartz Sand, pH, Ionic Strength, Isotherm Adsorption

\section{Introduction}

In large-scale livestock farming, the increasing consumption of various antibiotics including quinolones, tetracyclines, sulfonamides, etc. has become an irreversible trend around the world [1] [2]. However, most antibiotics cannot be completely assimilated into the animal body, and $40 \%$ to $90 \%$ of the antibiotic dose will be ex-

*The first author: Xiujiao Xu (1988), Female, Hebei, Master, is mainly engaged in the numerical simulation of underground water flow and solute transport in the environment.

"Corresponding author. 
creted with the feces and urine in the form of precursor or metabolite [3]. As a result, the final antibiotics in animal faeces may reach tens of $\mathrm{mg} / \mathrm{kg}$ or more [4] [5], which finally go into the soil environment through the application of agricultural manure. Relevant studies indicate that the amount of antibiotics entering into the soil is comparable to the pesticide application rate [6]. Antibiotics not only affect soil fertility and the safety of agricultural products, but also pollute water by surface runoff and leaching, ultimately threatening animal and human health. Nowadays, the antibiotics directly entering into the soil environment through the application of agriculture manure have become a potential risk of environmental pollution [7]-[9]. Therefore, the antibiotic pollution has become an important environmental organic pollutant and meanwhile a hotspot in environmental science.

Ciprofloxacin (CIP) is a quinolone antibiotic widely used in livestock and poultry breeding industry because of its broad-spectrum antibacterial and efficiency. Along with its popularization, various environmental problems also appear, and have attracted more and more attentions from domestic and foreign scholars. However, current research on CIP mainly focuses on the clinical application of animal diseases, animal in vivo pharmacokinetics, drug residues in animal products, etc. [10]-[13], but seldom concerns the transport behaviors of CIP after it enters into the environment from the perspective of ecological security. There are less research on CIP adsorption and transport in the soil, and the situation is even worse in China.

Prior to this study, the CIP adsorption characteristics in the soil at different $\mathrm{pH}$ and ionic strength have been studied using the measurement methods including HPLC, MS, GC, etc. [14]. However, due to the restriction of current laboratory conditions, the samples were analyzed by UV spectrophotometer, and the experimental data were not ideal. There were probably two reasons: Firstly, the soil contained a lot of organic matter that interfered with the analytical measurement, so that the peaks were not obvious at $273 \mathrm{~nm}$ wavelength under UV detection conditions; secondly, the mineral composition in the soil, introduced by inappropriate centrifugal filtration, led to the filtrate turbidity, which resulted in inaccurate measurements. At the beginning, it was suspected that the filtration operation was not good enough, but the experimental data were not improved even with qualitative and quantitative filter paper. Further, after EDTA-2Na salt was added in the filtrate as reagent, the results of UV spectral scanning were not improved yet. Considering UV could not accurately measure the CIP in the soil, ICP was tried to measure the CIP in the soil indirectly through measuring the zinc content by creating CIPzinc complexes in the sample. However, the complex concentration was too low to produce obvious precipitation, and no meaningful experimental data were obtained.

Therefore, considering the complexity of the soil system, in order to analyze the adsorption characteristics of ciprofloxacin under a single factor, the quartz sand of low reactivity was taken as a medium to explore the CIP absorption in quartz sand at different $\mathrm{pH}$ and ionic strength, and the mixed displacement experiments were carried out indoors to demonstrate the vertical transport characteristics of CIP in the soil profile. This study can provide a scientific basis for further revealing adsorption mechanism in the soil and the ecological risk assessment and management, and meanwhile plays an important role in the rational use of ciprofloxacin in animal husbandry.

\section{Materials and Methods}

\subsection{Instruments and Reagents}

Instruments: Oscillator THZ-92A, Haibo Motion Industries Limited Medical Equipment Factory; Centrifuge TDL-4A, Heidfeld Pradesh Analytical Instruments Co., Ltd.; UV spectrophotometer, Beijing Lebo Tektronix Instrument Co., Ltd.; pH meter PHS-3C, Shanghai Jing Branch Instrument Co., Ltd.; Soil column with a height of $10 \mathrm{~cm}$ and an inner diameter of $5 \mathrm{~cm}$, own product made of plexiglass; Peristaltic pump, BT100-1F, Baoding Lange cross-flow pump, Ltd.; Automatic part collection, BSZ-100, Shanghai Huxi Analytical Instrument Factory.

Reagents: CIP reference (purity 98.0\%, Tokyo Kasei Kogyo Co., Ltd.); other chemical reagents (analytical grade); test water, crystal-D ultrapure water.

\subsection{Test Materials}

Quartz sand is a kind of hard and wear-resistant quartzte mineral with stable chemical properties. Its main mineral component is $\mathrm{SiO}_{2}(99.5 \mathrm{wt} \%)$, along with tiny iron oxide ( $\left.<0.02 \mathrm{wt} \%\right)$, clay, mica and organic impurities. 
Quartz sand is milky white or colorless translucent solid with the relative density of 2.65. It is insoluble in acid but slightly soluble in $\mathrm{KOH}$ solution. The quartz crystals own porous structure, which can absorb various particles or molecules. In general, the adsorption and chemical properties of $\mathrm{SiO}_{2}$ surface are mainly determined by the surface hydroxyl groups, and CIP is just adsorbed on the surface of the medium by forming hydrogen bonds with hydroxyl groups [15]. Before each experiment, the quartz sand was sieved over 20 - 30 mesh sieve, and then soaked in $0.01 \mathrm{~mol} / \mathrm{L} \mathrm{NaOH}$ for $24 \mathrm{~h}$ and $0.01 \mathrm{~mol} / \mathrm{L} \mathrm{HCl}$ for $24 \mathrm{~h}$ successively to remove the metal oxide on the surface. After soaked in $\mathrm{NaOH}$ or $\mathrm{HCl}$ solution, the quartz sand was washed with distilled water until the $\mathrm{pH}$ was close to neutral. After dried at $105^{\circ} \mathrm{C}$, the spare test materials were obtained [16].

\subsection{Experimental Methods}

\subsubsection{Adsorption Isothermal Experiment}

In this experiment, eighteen $50 \mathrm{~mL}$ centrifuge tubes were divided into three groups, and $1 \mathrm{~g}$ sample of quartz sand was added into each tube respectively. Then, CIP-KCl solutions of different concentrations were prepared using $0.01 \mathrm{~mol} / \mathrm{L} \mathrm{KCl}$ solution as supporting electrolyte, and the CIP concentrations in the same group were 0 , $10,20,30,40,50 \mathrm{mg} / \mathrm{L}$ respectively. $\mathrm{pH}$ value of the three groups was adjusted to 5, 6 and 7 respectively, and $20 \mathrm{~mL}$ CIP solution with different $\mathrm{pH}$ was added into each centrifuge tube respectively according to grouping requirements. The solution was sealed and shaken at $25^{\circ} \mathrm{C}$ for $24 \mathrm{~h}$, and then centrifuged. The supernatant was removed by filtration and the filtrate was diluted by 5 times. The CIP concentration of the supernatant was measured by UV spectrophotometer, and then the adsorption amount was calculated. Three replicates were done for the above operations, and the blank (CIP solution without soil) was set. In order to avoid oscillations during the photo degradation of antibiotics, the whole process was carried out in the dark.

\subsubsection{Effects of Ionic Strength on CIP Adsorption}

Monovalent metal ions such as $\mathrm{Na}^{+}$and $\mathrm{K}^{+}$have an impact on the adsorption by competing adsorption sites with antibiotics of cationic state or zero valence state [17]. With the same experimental method described above, the pH of CIP was keep as 6 and the ionic strength were $0.01,0.05$ and $0.1 \mathrm{~mol} / \mathrm{L}$, respectively. Then, the CIP concentration in solution was measured and the adsorption capacity was calculated. The measurements above were repeated three times.

\subsubsection{Soil Column Outflow Experiments}

To analyze the effects of pH and ionic strength on CIP transport in quartz sand, the soil column outflow experiments were carried out. The experiments were conducted indoors in one-dimensional saturated soil column with a height of $10 \mathrm{~cm}$ and a diameter of $5 \mathrm{~cm}$. The washed and dried quartz sand was filled into the soil column in 5 times, and the soil column was compacted by plastic compactor each time to ensure uniform distribution of quartz sand particles. Meanwhile, some cotton wool was added to the higher and lower ends of each interface in the soil column in order to prevent quartz sand particles from clogging the water hole. At the each end there was a layer of filter paper which ensured that the solution could be uniformly penetrated into the quartz sand [18]. Taking a beaker and a peristaltic pump as the water supply devices, the peristaltic pump at a fixed speed pushed the background solution (KCL solution concentrations of $0.01 \mathrm{~mol} / \mathrm{L}, 0.05 \mathrm{~mol} / \mathrm{L}$ and $0.1 \mathrm{~mol} / \mathrm{L}$ ) with adjusted $\mathrm{pH}$ (5, 6 and 7) from the bottom slowly into the soil column in order to drain the air in soil column. When the soil column was saturated and the steady flow field was formed, with the pulse input method, $1.0 \mathrm{pv}$ CIP solution of $50 \mathrm{mg} / \mathrm{L}$ was input from the upper soil column, and then the soil column was rinsed with $\mathrm{KCl}$ background solution. The auto collector collected the flow fluid until the observed CIP concentration tended to zero in the flow fluid (two replicates for each experiment). By the way, $p v=v t / l$ is a dimensionless time, where $v$ represents the pore water velocity $(\mathrm{cm} / \mathrm{h}), t$ represents time $(\mathrm{h})$, and $l$ represents the soil column length $(\mathrm{cm})$.

\subsection{Data Processing}

\subsubsection{Adsorption Amount}

$$
S=\frac{\left(C-C_{0}\right) V}{m}
$$

where $S$ is the CIP equilibrium adsorption on quartz sand (mg/kg), $C_{0}$ is the CIP concentration in the added solu- 
tion $(\mathrm{mg} / \mathrm{L}), C$ is the CIP concentration in the balanced solution $(\mathrm{mg} / \mathrm{L}), V$ is the equilibrium liquid volume $(\mathrm{L})$, and $m$ is the mass of added quartz sand $(\mathrm{kg})$.

\subsubsection{Fitting Equation}

Empirical formulas of Langmuir and Freundlich isothermal adsorption curves can well fit the adsorption experimental data. By fitting equations, each coefficient and certainty coefficient can be determined, and thus the CIP adsorption capacity on the quartz sand can be analyzed [19].

Langmuir equation:

$$
S=\frac{K_{L} S_{m} C}{1+K_{L} C}
$$

Freundlich equation:

$$
\mathrm{S}=K_{F} C^{n}
$$

In the above equations, $S_{m}$ represents the maximum CIP adsorption amount on the quartz sand $(\mathrm{mg} / \mathrm{kg}), K_{L}$ represents the affinity of the quartz sand to CIP adsorption, $K_{F}$ is a constant, and $\mathrm{n}$ is the adsorption reaction order (usually less than 1).

\section{Results and Discussion}

\subsection{Static Adsorption}

\subsection{1. pH Influence on the Adsorption of CIP on Quartz Sand}

As Figure 1 shows, in the $\mathrm{pH}$ range of 5.0 to 7.0, the higher $\mathrm{pH}$ leads to the lower CIP adsorption amount. $\mathrm{pH}$ generally affects the adsorption behaviors of adsorbent in water environment by changing the state of the soil surface and the morphology of solute molecules in solution. The adsorption on quartz sand mainly depends on its surface properties, and the surface reactivity mainly concerns about the surface hydroxyl groups. The CIP contains $-\mathrm{NH}_{3}$ and $-\mathrm{COOH}$, which can combine with the $\mathrm{H}^{+}$and $\mathrm{OH}^{-}$in solution respectively, so that the CIP can exist in solution in three forms including cation, zwitterion and anion. According to the pKa value of CIP $\left(\mathrm{pKa}_{1}=6.10, \mathrm{pKa}_{2}=8.70\right)$ [20], the proportion of CIP in different forms can be calculated as shown in Figure 2 . When $\mathrm{pH}$ is less than pKa1, $-\mathrm{NH}_{3}$ in CIP can form $\mathrm{CIPH}^{+}$by combining with $\mathrm{H}^{+}$in solution, which benefits the CIP adsorption on the negatively-charged surface of quartz sand. In the $\mathrm{pH}$ range of 4 to 6 , most of CIP is cationic. With $\mathrm{pH}$ increasing, the CIP cations in the solution decrease, while neutral ions gradually increase. When $\mathrm{pH}=7.5$, the CIP almost exists in electrically neutral zwitterionic form (+ - 0) [21], and the CIP cationic groups can combine with negatively charged cations on the CIP surface by cation exchange. With the proportion decline of positive charge in CIP, the proportion of zwitterionic molecules gradually increases, so that the adsorp-

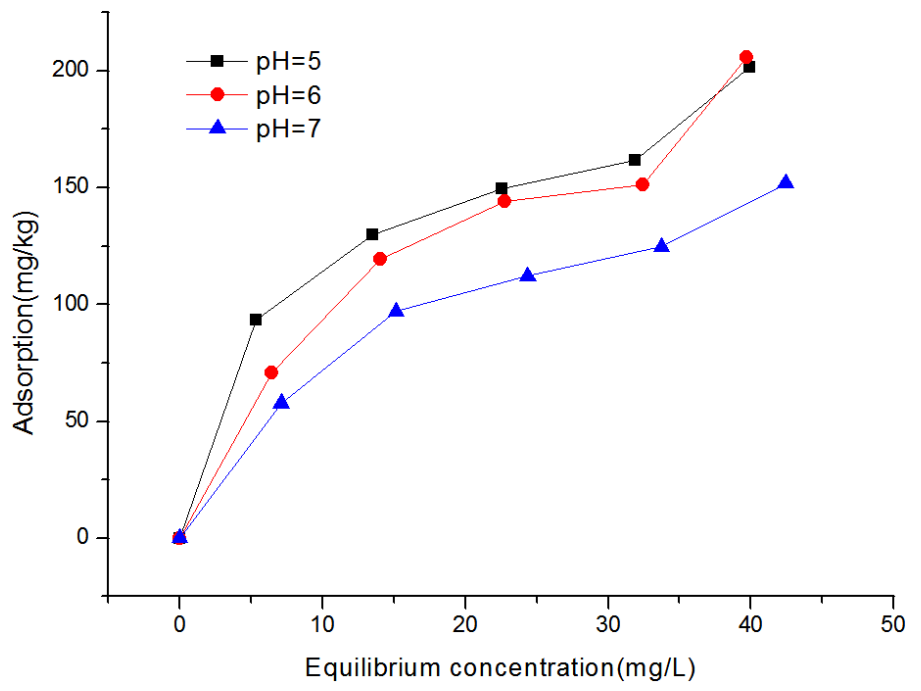

Figure 1. The adsorption isothermal of CIP at different $\mathrm{pH}$ in quartz sand. 




Figure 2. Speciation of CIP at different $\mathrm{pH}$ [20].

tion capacity of CIP on the quartz sand gradually weakens. Thus, the absorption of CIP on the quartz sand proceeds mainly through cation exchange adsorption [22]. Meanwhile, the adsorption of CIP on quartz sand also relates to its own $\mathrm{pH}$ to some extent. Under acidic conditions, cation exchange effect is helpful to adsorb CIP on quartz sand, the CIP cations can combine with negatively charged quartz sand by cation exchange action; while under neutral conditions, the electrically neutral CIP molecules are absorbed on the quartz sand mainly by Van der Waals force.

\subsubsection{Influence of Ionic Strength on the Adsorption of CIP on Quartz Sand}

Figure 3 shows the comparative adsorption of CIP at different ionic strength $(0.01 \mathrm{~mol} / \mathrm{L}, 0.05 \mathrm{~mol} / \mathrm{L}, 0.1 \mathrm{~mol} / \mathrm{L})$ when $\mathrm{pH}=6$. As can be seen, the bigger the ionic strength is, the lower the adsorption amount of CIP on quartz sand is. Probably, the increase of ionic strength makes the concentration of the competitive cation $\mathrm{K}^{+}$on quartz sand increase, so that a large amount of negative charge points are occupied by $\mathrm{K}^{+}$on quartz sand surface. Consequently, ions adsorbed by adsorption point tend to be saturated, which reduces the electrical adsorption ability of the quartz sand and thus the CIP adsorption amount on the quartz sand.

Table 1 shows the $\mathrm{K}_{\mathrm{d}}$ value distribution coefficient of CIP in quartz sand at different ionic strength when $\mathrm{pH}$ $=6\left(\mathrm{~K}_{\mathrm{d}}\right.$ is the ratio of CIP absorption amount per unit mass of soil to the CIP concentration in equilibrium solu-

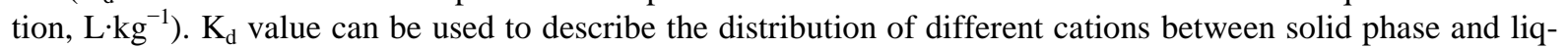
uid phase in soil. Low $K_{d}$ value means that most CIP exists in the soil solution with potential activity and transport ability, while high $K_{d}$ value indicates that there is a strong affinity between CIP and the surface of soil particles so that CIP can be easily adsorbed by the surface of soil particles.

As shown in Table $1, K_{d}$ value on the quartz sand gradually decreases with the increase of CIP concentration in equilibration solution. Meanwhile, as ionic strength of the solution increases, $K_{d}$ value of CIP on quartz sand decreases. Through the analysis above, the adsorption of CIP on quartz sand proceeds mainly by the ion exchange between the negatively charged quartz sand surface and CIP cationic groups. With the increase of cation concentration in quartz sand solution, they compete with CIP cation active groups for active adsorption sites, so that the adsorption amount of CIP decreases. With the increase of initial CIP concentration, $\mathrm{K}_{\mathrm{d}}$ value of CIP on quartz sand decreases, indicating the decrease of absorption rate in balanced solution. In low equilibrium concentration, specific adsorption with high energy absorption sites dominates the adsorption process. With the increase of CIP concentration in balanced solution, specific adsorption sites are gradually occupied and non-specific adsorption increases. As a result, the adsorption capacity of quartz sand relatively reduces, and $\mathrm{K}_{\mathrm{d}}$ value of CIP decreases [23]-[24].

\subsubsection{Data Fitting for the CIP Adsorption on Quartz Sand}

Figure 4 shows the fitting results of the CIP adsorption on the quartz sand by the Langmuir and Freundlich adsorption isothermal models. Obviously, the CIP adsorption characteristics on the quartz sand are nonlinear, and the obtained adsorption parameters are shown in Table 2. In the fitting process by Langmuir equation, the correlation coefficient $R^{2}$ is larger than 0.806 , while the correlation coefficient $R^{2}$ is larger than 0.886 by Freundlich equation. Meanwhile, $n$ is between 0.3 and 0.6 , which shows that the adsorption isotherms are S-type adsorption 


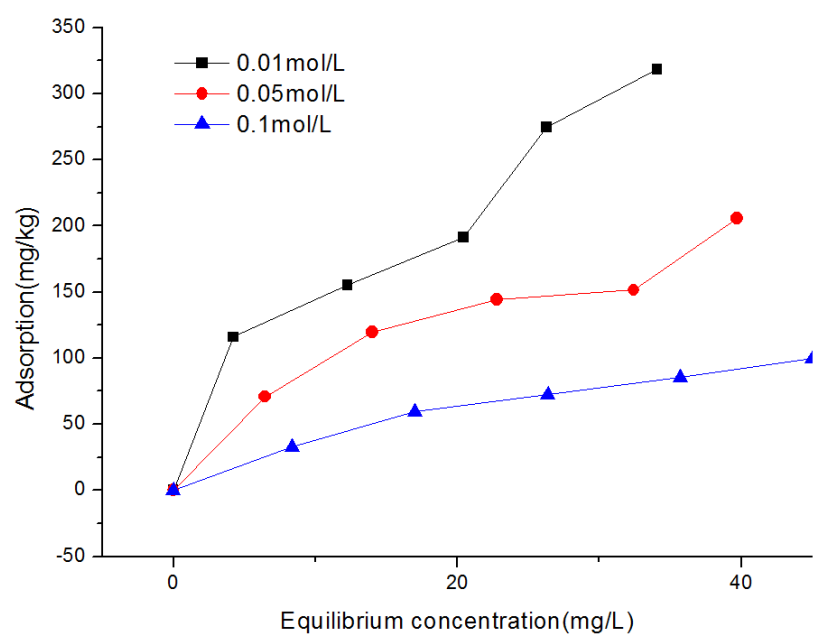

Figure 3. The adsorption isothermal of ciprofloxacin at different ionic strength in quartz sand.

Table 1. The distribution coefficient of CIP at different ionic strength in quartz sand when $\mathrm{pH}=6$.

\begin{tabular}{ccccccc}
\hline & \multicolumn{5}{c}{ Concentration $(\mathrm{mg} / \mathrm{L})$} \\
\cline { 2 - 7 } Ionic strength (mol/L) & 10 & 20 & 30 & 40 & 50 \\
\hline 0.01 & 15.7 & 14.5 & 8.7 & 10.5 & 9.3 \\
0.05 & 11.0 & 8.5 & 6.3 & 4.7 & 5.2 & 3.9 \\
\hline 0.1 & 2.9 & 3.4 & 4.4 & 3.7 & \\
\hline
\end{tabular}


Figure 4. Adsorption curves of ciprofloxacin at different $\mathrm{pH}$ and ionic strength. 
Table 2. The adsorption isotherm parameters of CIP at different $\mathrm{pH}$ and ionic strength.

\begin{tabular}{ccccccccc}
\hline & & \multicolumn{3}{c}{ Freundlich } & \multicolumn{3}{c}{ Langmuir } \\
\cline { 3 - 8 } & Ionic strength (mol/L) & $K_{F}$ & $n$ & $\mathrm{R}^{2}$ & $S_{m}$ & $K_{L}$ & $\mathrm{R}^{2}$ \\
\hline 5 & 0.05 & 48.8 & 0.368 & 0.929 & 223.3 & 0.111 & 0.842 \\
& 0.01 & 37.9 & 0.592 & 0.886 & 579.7 & 0.032 & 0.806 \\
6 & 0.05 & 27.5 & 0.527 & 0.901 & 286.2 & 0.047 & 0.876 \\
& 0.1 & 10.0 & 0.603 & 0.985 & 173.8 & 0.028 & 0.989 \\
7 & 0.05 & 23.8 & 0.488 & 0.960 & 207.4 & 0.053 & 0.950 \\
\hline
\end{tabular}

Note: $\mathrm{R}^{2}$ is the certainty coefficient, which represents the fitness of fitting values and measured values. The closer to 1 means the better fitness.

isotherms and that the adsorption type is nonlinear adsorption. Generally, this adsorption type can be attributed to the competition between water molecules and CIP molecules for adsorption sites on the soil surface. At low CIP concentrations, the affinity of CIP to the soil is weaker than that to the water phase; while with the increase of CIP concentration, its affinity to the soil increases, so that the adsorption amount increases. In contrast, if " $n>1$ ", the adsorption isotherm belongs to L-type adsorption isotherms. At low concentrations, the affinity of CIP to the soil is stronger than that to the water phase; while with the increase of CIP concentration, its affinity to the soil decreases, so that the adsorption weakens [25]. $K_{F}$ and $K_{L}$ can be used to evaluate the adsorption affinity of CIP on the quartz sand. As can be seen from Table 2, at a constant $\mathrm{pH}$, the higher the ion intensity is, the smaller the affinity of CIP to the soil is, and thus the greater the fitted maximum adsorption amount is. The result is consistent with the experimental result. Under the same ionic strength, for Freundlich model, the higher the $\mathrm{pH}$ is, the smaller the affinity of CIP to the soil is, which is consistent with experimental result; while the adsorption isotherm fitting result by Langmuir model is not consistent with the experimental result. That is to say, Langmuir equation has certain limitations to fit the adsorption isotherm of competitive ions.

Adsorption reaction is the most important process during the transport and transformation of CIP in quartz sand, which can reflect the degree of interaction between CIP and the quartz sand and predict the stability of CIP in the quartz sand. Generally, the CIP is very stable if it can be strongly adsorbed by quartz sand, so that it is easier to accumulate in quartz sand instead of migration. In contrast, if the CIP cannot be absorbed and fixed easily on quartz sand, it is easy to be transported into surface water or groundwater under eluviations effect. The above results show that low $\mathrm{pH}$ and low ionic strength are conductive to the fixing of CIP in the soil, which eliminate its migration with the water.

\subsection{Soil Column Transport Experiments}

\subsubsection{The Breakthrough Curves of CIP Transport in Quartz Sand at Different pH}

Figure 5 shows the breakthrough curves of CIP in quartz sand at different $\mathrm{pH}(5,6,7)$ when the ionic strength is $0.05 \mathrm{~mol} / \mathrm{L}$. The CIP solution of $\mathrm{pH}=7$ starts to outflow when $\mathrm{pv}=0.47$, then the CIP solution of $\mathrm{pH}=6$ starts to outflow when $\mathrm{pv}=0.51$, and finally the CIP solution of $\mathrm{pH}=5$ starts to outflow when $\mathrm{pv}=0.54$. The greater the $\mathrm{pH}$ is, the sooner the outflow is. The reason is that the greater $\mathrm{pH}$ usually weakens the CIP adsorption capacity on quartz sand, so that the CIP can outflow very fast, with a stronger migration. Prior to $1.0 \mathrm{pv}$, it is the adsorption stage, followed by the desorption stage. When $\mathrm{pv}=1.0$, the backwash stage (adsorption-desorption phase) starts, and then the CIP concentration gradually increases. When $\mathrm{pv}=1.63$, the solution of $\mathrm{pH}=5$ reaches the maximum concentration, with a relative concentration $\mathrm{C} / \mathrm{C}_{0}$ of 0.88 ; when $\mathrm{pv}=1.56$, the solution of $\mathrm{pH}=6$ reaches the maximum concentration, with a relative concentration $\mathrm{C} / \mathrm{C}_{0}$ of 0.96 ; when $\mathrm{pv}=1.65$, the solution of $\mathrm{pH}=7$ reaches the maximum concentration, with a relative concentration $\mathrm{C} / \mathrm{C}_{0}$ of 0.89 . After that, the CIP concentration decreases rapidly. During this process, the solution of $\mathrm{pH}=7$ desorbs most rapidly, followed by the solution of $\mathrm{pH}=6$ and the solution of $\mathrm{pH}=5$. The higher the $\mathrm{pH}$ is, the smaller the adsorption amount is, and thus the greater the desorption amount is. In the transport process from solution outflow to the concentration being zero, the volume of the solution of $\mathrm{pH}=5$ is $2.50 \mathrm{pv}$, that of $\mathrm{pH}=6$ is $2.48 \mathrm{pv}$, and that of $\mathrm{pH}=7$ is $2.36 \mathrm{pv}$. The CIP starts to outflow earliest when $\mathrm{pH}=7$, followed by $\mathrm{pH}=6$ and $\mathrm{pH}=5$. This difference may be related to the different adsorption capacity of CIP at different $\mathrm{pH}$. The lower the $\mathrm{pH}$ is, the stronger the adsorption capacity is, and thus the longer the outflow time is. On the contrary, the greater the $\mathrm{pH}$ is, the shorter the outflow 


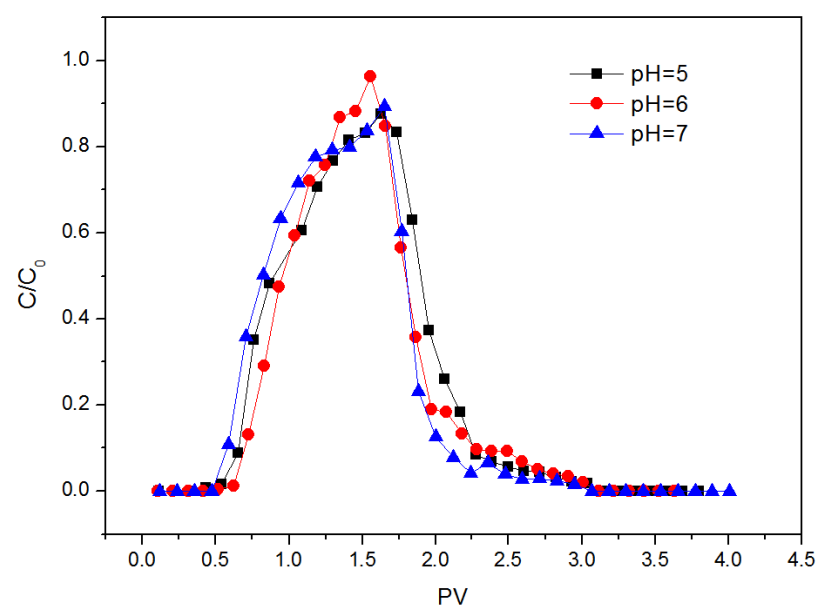

Figure 5. The breakthrough curves at different $\mathrm{pH}$ when the ionic strength is $0.05 \mathrm{~mol} / \mathrm{L}$.

time is. When $\mathrm{pH}=5$, the desorption phase has a long tail due to the slow desorption caused by large adsorption amount. Hence, if the soil is in alkaline environment, the CIP concentration in the soil will increase, and the CIP is easier to migrate in the soil, thus increasing the risk of groundwater contamination.

\subsubsection{The Breakthrough Curves of CIP Transport in the Quartz Sand at Different Ionic Strength}

Figure 6 shows the breakthrough curves of CIP in quartz sand at different ionic strength $(0.01,0.05,0.1 \mathrm{~mol} / \mathrm{L})$ when $\mathrm{pH}=6$. In the adsorption phase, the CIP adsorbs fast and outflows slowly at the ionic strength of 0.01 $\mathrm{mol} / \mathrm{L}$. The greater the ionic strength is, the faster the outflow is. The reason may be that a large number of $\mathrm{K}^{+}$ ions adsorbed on the quartz solid surface compete with CIP for the adsorption sites, resulting in the decrease of CIP adsorption amount on quartz sand and further delaying the outflow. In the desorption stage, at the ionic strength of $0.01 \mathrm{~mol} / \mathrm{L}$, CIP desorbs slowly, and there presents a tail. This process is a continuous and slow desorption process. With the ionic strength increasing, the desorption becomes fast, which promotes the transport of CIP in quartz sand. At the same time, when the ionic strength is large, a large number of $\mathrm{K}^{+}$ions occupy the negatively charged adsorption sites on the surface of medium, and the negatively charged adsorption sites tend to be saturated, which reduces the adsorption of CIP. In addition, the increase of ionic strength will enhance the ionic interaction in systems and reduce the ion activity coefficient, which decreases the effective concentration of CIP and accelerates its transport in the soil column. Hence, if the soil is contaminated by a high-salt wastewater, it will accelerate the desorption of CIP and promote its transport in the soil, thus polluting the groundwater.

\section{Conclusions}

In summary, the CIP adsorption and transport characteristics in quartz sand were investigated at different $\mathrm{pH}$ and ionic strength conditions, and some conclusions can be drawn as follows:

1) The adsorption of CIP on the quartz sand belonged to cation exchange adsorption, and the variations of $\mathrm{pH}$ and ion morphology had a significant influence on the adsorption capacity.

2) When $\mathrm{pH}$ was in the range from 5 to 7 , the higher the $\mathrm{pH}$ was, the lower the adsorption capacity of CIP on quartz sand was; the greater the ionic strength was, the lower the CIP adsorption amount on quartz sand was. That was to say, the low $\mathrm{pH}$ and low ionic strength were in favor of CIP adsorption on quartz sand. With the increase of CIP concentration in balanced solution, the $\mathrm{K}_{\mathrm{d}}$ value of CIP in quartz sand decreased; meanwhile, with the increase of ionic strength in the solution, the $K_{d}$ value of CIP in quartz sand decreased.

3) The CIP adsorption on quartz sand could be described by the Langmuir and Freundlich equations, and the Freundlich equation fitted the adsorption isotherms better.

4) When $\mathrm{pH}$ was in the range from 5 to 7 , the higher the $\mathrm{pH}$ was, the faster the transport of the CIP on quartz sand was, and thus the earlier the outflow was. That was to say, the higher the $\mathrm{pH}$ was, the stronger the adsorption capacity was, and thus the shorter the outflow time was. Meanwhile, the increase of ionic strength would 


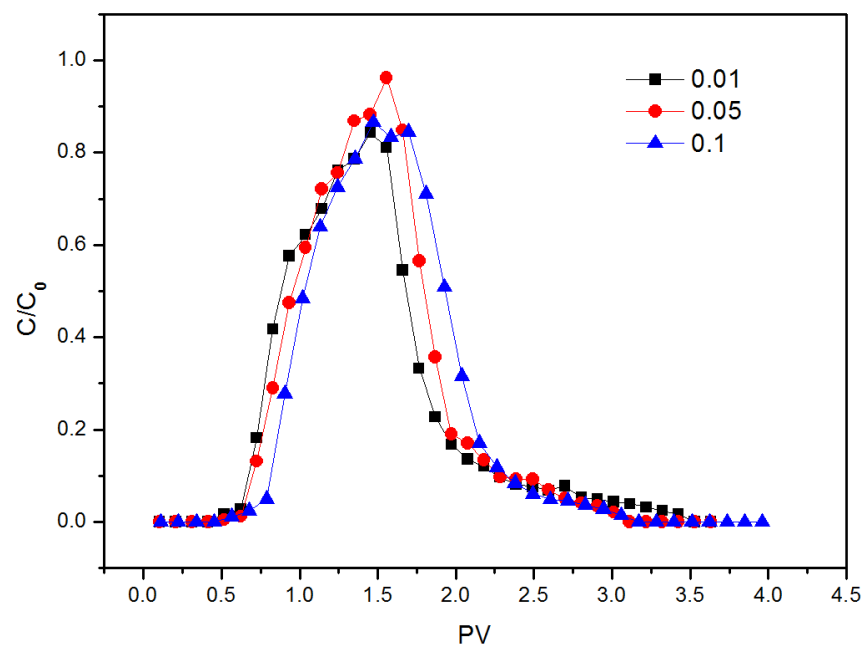

Figure 6. The breakthrough curves at different ionic strength when $\mathrm{pH}=6$.

reduce the absorption of CIP on quartz sand, which led to the faster transport and the earlier outflow. As a large number of $\mathrm{K}^{+}$ions occupied the negatively charged adsorption sites, the medium surface tended to be saturated, so that the adsorption capacity of CIP on quartz sand was reduced.

\section{Acknowledgements}

This study was accomplished with participation of the undergraduates innovative experimental group members Jiang-Long He, Yu Li, Zhao-Xi Fang, Xin Li and Ya-Kun Hu.

\section{Foundation Item}

National Natural Science Foundation of China (40771095), Qingdao people’s livelihood project (13-1-3-132-nsh).

\section{References}

[1] Kools, S.A.E., Moltmann, J.F. and Knacker, T. (2008) Estimating the Use of Veterinary Medicines in the European Union. Regulatory Toxicology and Pharmacology, 50, 59-65. http://dx.doi.org/10.1016/j.yrtph.2007.06.003

[2] Hou, F.L. (2003) Feed Additive Application Da Quan. China Agriculture Press, Beijing, 1-45.

[3] Cui, H. and Wang, S.P. (2012) Adsorption Characteristics of Ciprofloxacin in Ustic Cambosols. Chinese Journal of Environmental Science, 33, 2895-2900.

[4] Hu, X.G., Luo, Y., Zhou, Q.X., et al. (2008) Determination of Thirteen Antibiotics Residues in Manure by Solid Phase Extraction and High Performance Liquid Chromatography. Chinese Journal of Analytical Chemistry, 36, 1162-1166. http://dx.doi.org/10.1016/S1872-2040(08)60063-8

[5] Liu, X.C., Dong, Y.H. and Wang, H. (2008) Residues of Tetracyclines in Animal Manure from Intensive Farm in Jiangsu Provilace. Journal of Agro-Environment Science, 27, 1177-1182.

[6] Haller, M.Y., Müller, S.R., McArdell, C.S., et al. (2002) Quantification of Veterinary Antibiotics (Sulfonamides and Trimethoprim) in Animal Manure by Liquid Chromatography-Mass Spectrometry. Journal of Chromatography A, 952, 111-120. http://dx.doi.org/10.1016/S0021-9673(02)00083-3

[7] Halling-Sorensen, B., Nors Nielsen, S., Lanzky, P.F., et al. (1998) Occurrence, Fate and Effects of Pharmaceutical Substances in the Environment-A Review. Chemosphere, 36, 357-393. http://dx.doi.org/10.1016/S0045-6535(97)00354-8

[8] Jemba, P.K. (2002) The Potential Impact of Veterinary and Human Therapeutic Agents in Manure and Biosoilds on Plants Grown on Arable Land: A Review. Agriculture, Ecosystems and Environment, 93, 267-278.

[9] Kumar, K., Gupta, S.C., Chander, Y., et al. (2005) Antibiotic Use in Agriculture and Its Impact on the Terrestrial Environment. Advances in Agronomy, 87, 1-54. http://dx.doi.org/10.1016/S0065-2113(05)87001-4

[10] Andon, A., Martinez-Larranage, M.R., Diaz, M.J., et al. (1995) Pharmacokinetics and Residues of Enrofloxacin in 
Chickens. American Journal of Veterinary Research, 56, 502-506.

[11] Manceau, J., Gicque, M., Laurentie, M., et al. (1999) Simultaneous Determination of Enrofloxacin and Ciprofloxacin in Animal Biological Fluids by High-Performance Liquid Chromatography Application in Pharmacokinetic Studies in Pig and Rabbit. Journal of Chromatography B, 726, 175-184.

[12] Dosogne, H., Meyer, E., Sturk, A., et al. (2002) Effect of Enrofloxacin Treatment on Plasma Endotoxin during Bovine Escherichia coli Mastitis. Inflammation Research, 51, 201-205. http://dx.doi.org/10.1007/PL00000293

[13] Vancutsem, P.M., Babish, J.G. and Schwark, W.S. (1990) The Fluoroquinolone Antimicrobials: Structure, Antimicrobial Activity, Pharmacokinetics, Clinical Use in Domestic Animals and Toxicity. The Cornell Veterinarian, 80, 173186.

[14] Ye, X.Q., Liu, D.H. and Chen, J.C. (2005) Advances in the Rapid Detection of Antibiotics Residues in Dairy Products. Transactions of the CSAE, 21, 181-185.

[15] Yang, J.W., Xu, L.Z., Jiang, X., et al. (2005) Study on the Adsorption of Aluminum Ions on Silica by X-Ray Photoelectron Spectroscopy. Chinese Journal of Spectroscopy Laboratory, 22, 666-668.

[16] Chu, L.Y., Si, Y.B. and Zhou, D.M. (2011) Study on the Migration of Nano-Hydroxyapatite and Carrying Heavy Metals$\mathrm{Cu}$ in Quartz sand Column. Anhui Agricultural University, Hefei.

[17] Qi, H.M., Lu, L. and Qiao, X.L. (2009) Progress in Sorption of Antibiotics to Soils. Soils, 41, 703-708.

[18] Sun, Y.Y. and Xu, S.H. (2013) Characteristic of $\mathrm{Zn}^{2+} / \mathrm{Cd}^{2+} / \mathrm{NH}^{4+}$ Transport in Soils with Different $\mathrm{pH}$ Value and Ionic Strength. Transactions of the Chinese Society of Agricultural Engineering, 29, 218-227.

[19] Wang, K.L., Xu, S.H., Yang, Y.L., et al. (2011) Study on Zn and Cd Colloid-Affected Adsorption in Three Different Soils. Soils, 43, 239-246.

[20] Wu, T.X. (2012) Adsorption of Ciprofloxacin on Three Different Soils. Northern Environmental, 25, 54-56.

[21] Gu, C. and Karthikeyan, K.G. (2005) Sorption of the Antimicrobial Ciprofloxacin to Aluminum and Iron Hydrous Oxides. Environmental Science \& Technology, 39, 9166-9173. http://dx.doi.org/10.1021/es051109f

[22] Wu, T.X. (2009) Studies on the Adsorption of Antibiotics in Soil. Northwest Normal University, Lanzhou.

[23] Basta, N.T. and Tabatai, M.A. (1992) Effect of Cropping Systems on Adsorption of Metals by Soils: III. Competitive Adsorption. Soil Science, 153, 331-337. http://dx.doi.org/10.1097/00010694-199204000-00010

[24] Yu, S., He, Z.L., Huang, C.Y., Chen, G.C. and Calvert, D.V. (2002) Adsorption-Desorption Behavior of Copper at Contaminated Levels in Red Soils from China. Journal of Environmental Quality, 31, 1129-1139. http://dx.doi.org/10.2134/jeq2002.1129

[25] Zhang, W., Wang, W.Q., Wang, J.J. and Li, G. (2010) Journal of Sichuan Normal University, 33, 366-371. 
Scientific Research Publishing (SCIRP) is one of the largest Open Access journal publishers. It is currently publishing more than 200 open access, online, peer-reviewed journals covering a wide range of academic disciplines. SCIRP serves the worldwide academic communities and contributes to the progress and application of science with its publication.

Other selected journals from SCIRP are listed as below. Submit your manuscript to us via either submit@scirp.org or Online Submission Portal.
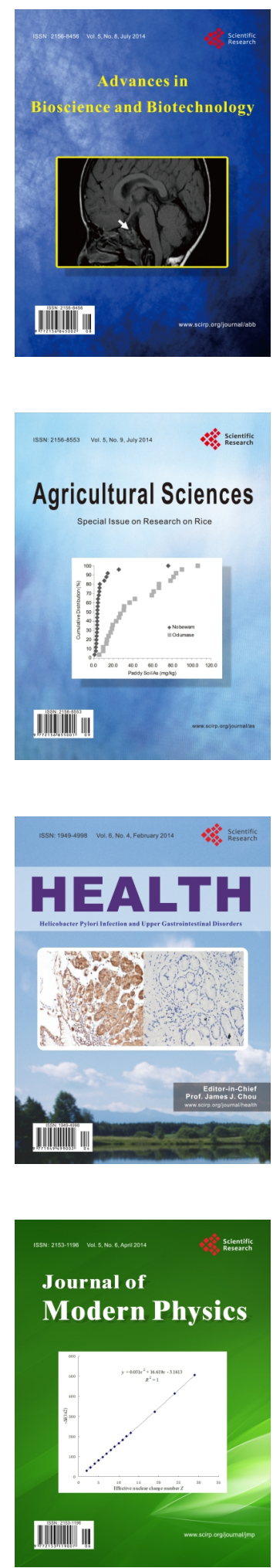
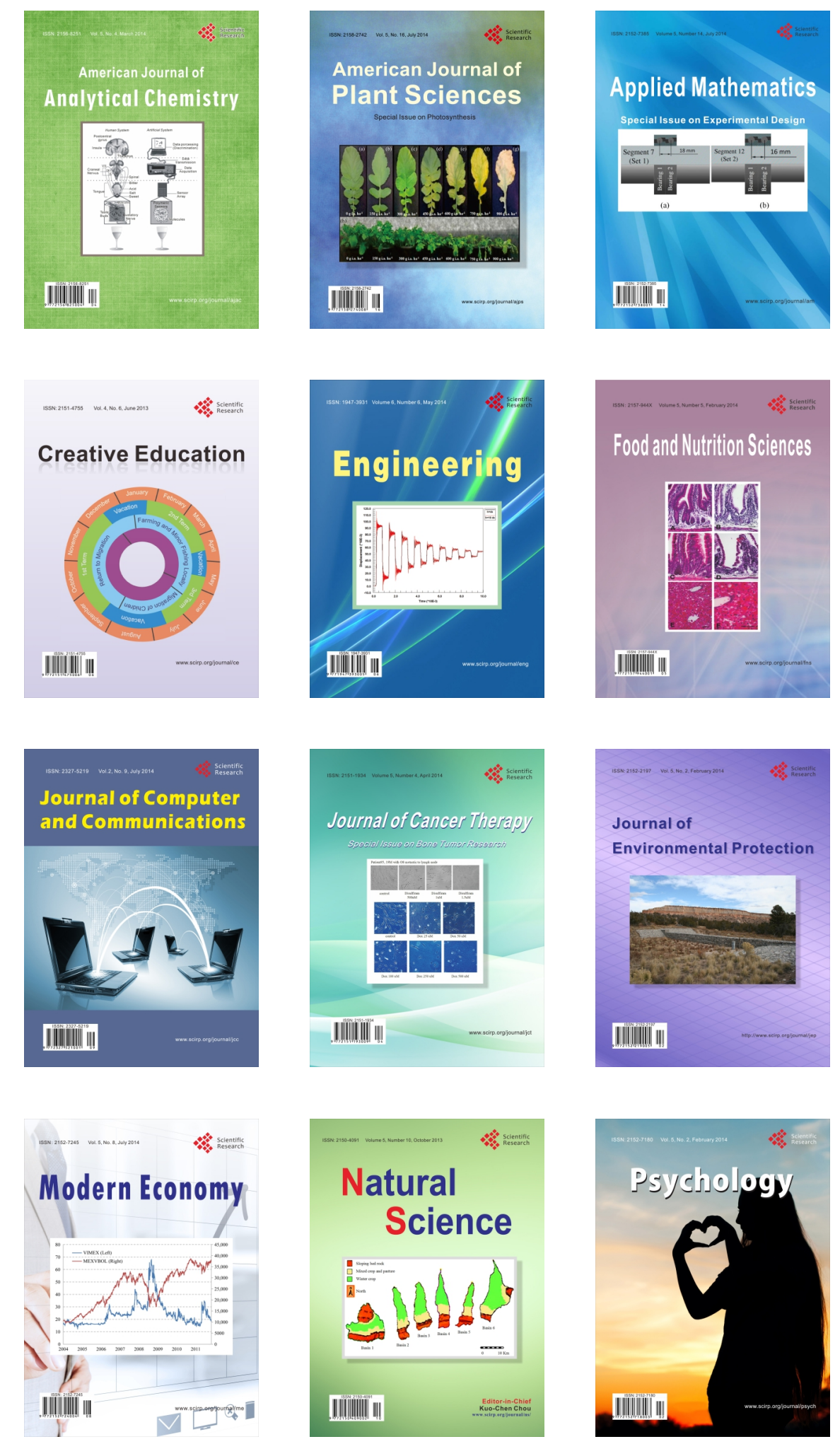